\title{
In memoriam: John Archibald Wheeler
}

Wheeler's early work in gravitation physics

\author{
Charles W. Misner
}

Published online: 6 March 2009

(C) The Author(s) 2009. This article is published with open access at Springerlink.com

John Archibald Wheeler (9 July 1911-13 April 2008) was a dominant figure in the GRG area from the time he taught his first course in General Relativity at Princeton in the spring of 1952 until his focus changed as he moved to Texas in 1976. But there were important connections between his earlier nuclear physics work and his later quantum fundamentals work. His earlier work, including the introduction of the S-matrix and the application of the nuclear liquid drop model to fission, enabled him to publish (and have read) daring ideas about the possibilities of implausible objects speculated to be consistent with the Einstein-Maxwell equations. His work on neutron star stability enabled him to combine the (then out-of-fashion) Einstein theory of gravity with his acknowledged expertise in nuclear forces, thus brushing GR with some of the respectability of nuclear physics. And then, during his daring conservativism phase of GR exploration, he supported a Ph.D. for Hugh Everett who had daring ideas about believing the Schrödinger equation at macroscopic levels. In this Wheeler was intrigued by the need to be able to talk about the wave function of the universe in order to study the very early Universe where he felt quantum mechanics could not be ignored. A focus on the fundamental role of the quantum was his major theme during his Texas years. For this journal I concentrate on his contributions to GRG, and in particular, those during the crucial early years when I was among the first of his many students in gravitation.

Two major events marked Wheeler's entry into the area of gravitation: his visiting semester in Leiden, and his group's publications in the July 1957 issue of Reviews of Modern Physics.

C. W. Misner $(\varangle)$

Gravitation Theory Group, Department of Physics,

University of Maryland, Room 4205D, College Park, MD 20742-4111, USA

e-mail: misner@umd.edu 
Invited to spend the spring and summer terms of 1956 as Lorentz Professor in Leiden, Holland, Wheeler brought Joseph Weber and graduate students Peter Putnam and myself with him. All four occupied one large office while Wheeler used another office to work on a long paper on nuclear physics. Weber had begun focusing on gravitational radiation the previous semester at the Institute for Advanced Study in Princeton, and continued this quest for the rest of his life, becoming the founding father of gravitational wave detection efforts, although misinterpreting his own progress years later. I worked with John to convert his qualitative ideas of "charge without charge", i.e., electric lines of force threaded though non-trivial spacetime topologies, into established mathematics. His "wormhole" sketch first appeared in a Phys. Rev. paper (v97, pp 511-536) submitted in September 1954, the month that I signed on as his Ph.D. advisee. By the time we went to Leiden in February 1956, I had informally learned (via math grad student friends) enough differential topology to relate his sketch to deRham cohomology. At Wheeler's suggestion I also read some of Lichnerowicz' 1955 text, learned the basics of the Einstein initial value equations, and realized that the existence of some solutions of the Einstein equations could be inferred from exact solutions of these initial value equations. This found several applications in Wheeler's group during the next few years as we sought to explore the range of physically interesting possible solutions of the Einstein equation, not just their mathematical properties. Another brief but significant activity in Leiden was John's bringing Tullio Regge there for a few weeks, which resulted in their initial development of the perturbation technology for the Schwarzschild metric. Decades of subsequent development led to the understanding of the "ring down" modes of oscillation of newly formed black holes and their gravitational radiation signatures.

Following the Chapel Hill conference ("GR1", organized by Cecile and Bryce DeWitt) in January 1957 the enormous scope of Wheeler's activity in 1955 and 1956, much by mail correspondence from Leiden, became visible in publications. The July 1957 issue of Reviews of Modern Physics (v 29, pp 351-546) contains, among others from this conference, nine articles from Wheeler's group. Among these papers were my Ph.D. thesis and that of Hugh Everett III. My work in Leiden with Wheeler, and also Regge's, were published separately (Ann. Phys. v2, pp 525-603, Phys. Rev. v108, pp 1063-1069) that year.

Particularly notable among the RMP papers, beyond Everett's thesis, were the papers by Lindquist and Wheeler, and by Weber and Wheeler. The Lindquist-Wheeler paper presents (in an approximation) what would now be described as a Friedmannlike closed universe which expands and recontracts, but whose only "matter" content was a finite number of identical black holes, symmetrically placed. This forcefully displayed Wheeler's "Geometrodynamics" idea: that the Einstein equations allow remarkably interesting systems to be constructed from pure vacuum, i.e., spacetime geometry only. I was able to supply an exact solution of the initial value equations at the moment of maximum expansion in these models, which permitted one calibration of the accuracy of the approximation used. This paper can also be considered the germ of the field, now active, of numerical relativity, as it contains the suggestion that a better approximation for the model would be provided when digital computers arrived at the point where they could evolve these exact initial conditions. For a still simpler model suggested there, this attempt was first made by Hahn and Lindquist 
in 1962 (Ann. Phys. NY, v29, p 304) but a satisfactory numerical evolution was not achieved until 1995 (Matzner et al., Science v270, pp 941-947). The Weber-Wheeler paper is interesting primarily because it gives evidence of the uncertainties at that time concerning whether gravitational waves are implied by Einstein's equations. Some relativists worried that the nonlinearities of the Einstein equations might make the waves found in linearized gravity misleading. Others worried that a binary star system, which has essentially two masses following geodesics and hence intrinsically unaccelerated, would not generate gravitational waves, even if a mechanically driven quadrupole oscillator might. The use of Einstein's and others' pseudotensors to measure gravitational energy was poorly understood, so by ignoring the conditions (later clarified) that asymptotically Minkowski coordinates are essential they cast doubt on the energy content of gravitational waves. The Weber-Wheeler paper is a step toward clarifying some of these issues.

Wheeler's gravitational activity continued at nearly this pace through the next two decades. Some of his students went on to form important groups of their own: Misner and Brill at Maryland, Thorne at Caltech, Geroch and Wald (recruited by Chandrasekhar) at Chicago, and Unruh at Vancouver. Others joined existing groups such as Syracuse or Austin. When Wheeler left Princeton for Texas, one prior student (Peter Putnam) organized that a book of letters ("Family Gathering") be written to John. Ninety-eight "students" responded, most having had gravitational interactions with him, including some who were never formally his students such as Aage Bohr, James Hartle, Roger Penrose, Larry Smarr. Some, such as James York and Remo Ruffini, had spent extended periods in Princeton as faculty members in Wheeler's group. Others such as Christodoulou, Teitelboim (now Bunster) and Ruffini returned to important positions in their homelands. But all were enthusiastic about their experience in seeing how Wheeler could focus energies on important questions ripe for progress, and on care and effort in explaining ideas to the broader physics community.

Open Access This article is distributed under the terms of the Creative Commons Attribution Noncommercial License which permits any noncommercial use, distribution, and reproduction in any medium, provided the original author(s) and source are credited. 